\title{
Association of Geriatric Syndromes with Urinary Incontinence according to Sex and Urinary-Incontinence-Related Quality of Life in Older Inpatients: A Cross-Sectional Study of an Acute Care Hospital
}

\author{
Kyoung Jin Kim', Jinyoung Shin', Jaekyung Choi '**, Jae-Min Park², Hyoung Keun Park³, Jongmin Lee, Seol-Heui Han \\ 'Department of Family Medicine, Konkuk University Medical Center, Konkuk University School of Medicine, Seoul, Korea \\ ${ }^{2}$ Department of Family Medicine, Gangnam Severance Hospital, Yonsei University College of Medicine, Seoul, Korea \\ ${ }^{3}$ Department of Urology, Konkuk University Medical Center, Konkuk University School of Medicine, Seoul, Korea \\ ${ }^{4}$ Department of Rehabilitation Medicine, Konkuk University Medical Center, Konkuk University School of Medicine, Seoul, Korea \\ ${ }^{5}$ Department of Neurology, Konkuk University Medical Center, Konkuk University School of Medicine, Seoul, Korea
}

Background: Geriatric syndromes are associated with morbidity and poor quality of life (QOL). Urinary incontinence (UI) is one of the most prevalent geriatric syndromes. However, there is little research on the association of UI and UI-related QOL with other geriatric syndromes. We investigated the relationship between geriatric syndromes and UI according to gender and UI-related QOL among older inpatients.

Methods: This study was conducted among 444 older inpatients (aged 65 years and older) between October 2016 and July 2017. We examined geriatric syndromes and related factors involving cognitive impairment, delirium, depression, mobility decline, polypharmacy, undernutrition, pain, and fecal incontinence. UI-related QOL was assessed using the International Consultation on Incontinence Questionnaire-Short Form. Multiple logistic regression analysis was used to evaluate these associations.

Results: Geriatric syndromes and related factors were associated with UI. Mobility decline (odds ratio [OR], 4.16; 95\% confidence interval [CI], 2.29-7.56), polypharmacy (OR, 3.35; 95\% CI, 1.89-5.92), and pain (OR, 6.80; 95\% CI, 3.53-13.09) were related to UI in both genders. Especially, delirium (OR, 7.55; 95\% CI, 1.61-35.44) and fecal incontinence (OR, 10.15; 95\% CI, 2.50-41.17) were associated with UI in men, while cognitive impairment (OR, 4.19; 95\% CI, 1.14-15.44) was significantly associated with UI in women. Patients with depression were more likely to have poor UI-related QOL (OR, 8.54; 95\% CI, 1.43-51.15).

Conclusion: UI was associated with different geriatric syndromes and related factors according to gender. Care for patients with depression, related to poor UI-related QOL, should be considered in primary care to improve the UIrelated QOL of these individuals.

Keywords: Urinary Incontinence; Geriatric Syndrome; Older Adults; Inpatients

Received: January 26, 2018, Revised: March 5, 2018, Accepted: June 16, 2018

${ }^{*}$ Corresponding Author: Jaekyung Choi https://orcid.org/0000-0002-0875-7505

Tel: +82-2-2030-7683, Fax: +82-2-2030-7748, E-mail: cjk@kuh.ac.kr 


\section{INTRODUCTION}

Geriatric syndromes are highly prevalent, multifactorial, and associated with substantial morbidity and poor health outcomes. Geriatric syndromes include delirium, cognitive impairment, geriatric depression, fecal incontinence, mobility decline, and urinary incontinence (UI). ${ }^{1)}$ Known risk factors of geriatric syndromes include polypharmacy, undernutrition, and pain. ${ }^{2)}$ Among the geriatric syndromes, UI is one of the most prevalent, ${ }^{1)}$ and is a widespread health concern among older adults. ${ }^{3)}$ UI substantially influences individuals' quality of life (QOL), particularly their physical, social, and psychological health status, as well as their morbidity and mortality. ${ }^{4)}$ As the elderly population grows, the number of older adults with UI is increasing along with the cost of healthcare. $^{5)}$

The reported risk factors of UI include age, gender, number of childbirths, previous hysterectomy or prostatectomy, obesity, various medical problems (e.g., infection, sores, falls, fractures, physical impairment), and chronic disease (e.g., stroke, benign prostate hyperplasia, and arthritis)..$^{6-10)}$ Mental problems such as low self-esteem, depression, and cognitive impairment are also associated with UI. ${ }^{4)}$ Among older adults with impaired overall function, UI is more likely to occur not only because of underlying diseases but also because of the various functional disabilities that patients have at the time of hospital admission. ${ }^{7,11)}$ Older adults who visit acute care hospitals are more likely to exhibit geriatric syndromes, such as multiple chronic diseases, impaired mobility, higher levels of dependency, and impaired cognition. These patients not only are at greater risk for a lengthy hospital stay with functional decline, ${ }^{12)}$ but also deteriorating QOL. ${ }^{13)}$ Despite the dramatic influence of UI in older patients, this syndrome has remained understudied during their stay at acute care hospitals. Specifically, there is little research on the association of UI and UI-related QOL with other geriatric syndromes in acute care hospital settings. Moreover, most studies have focused on UI in women, so there is insufficient knowledge of incontinence in men. Consequently, we investigated the relationship between geriatric syndromes and UI, and how these associations differed by gender and UI-related QOL among older inpatients.

\section{METHODS}

\section{Study Subjects}

We enrolled 444 patients aged 65 years or older who were hospitalized between October 2016 and July 2017 in an acute care hospital. We compared the general characteristics of the two groups: with and without UI. Patients with UI were asked to complete the International Consultation on Incontinence Questionnaire-Short Form (ICIQ-SF) to identify the frequency, amount, and severity of UI and its impact on QOL. ${ }^{14)}$ We also examined geriatric syndromes and some of their known related factors. The institutional review board (IRB) of Konkuk University Medical Center approved this study (IRB approval no., KUH11701347) and complied with the tenets of the Declaration of
Helsinki. Written informed consent was obtained from each participant before or at registration.

\section{Measurements}

\section{1) Demographics and comorbidities}

Demographic data, such as age, gender, insurance, body mass index (underweight, normal, overweight, and obese), and length of hospital stay, were obtained through a review of patients' electronic medical records. Past medical history of hypertension, diabetes, stroke, and experience of any surgery was also obtained by reviewing these medical records based on physicians' diagnosis or prescription.

\section{2) Urinary incontinence}

Patients were identified as having UI if they responded affirmatively to the following diagnostic question: ${ }^{15)}$ "Have you experienced accidental urine leakage in the last month?"

\section{3) International Consultation on Incontinence Questionnaire-Short Form}

The ICIQ-SF was completed by patients with UI to assess the frequency, amount, and severity of UI as well as its impact on QOL. ${ }^{14)}$ This instrument comprises four subscales: (1) frequency ("How often do you leak urine?"; 0, never; 1, once a week; 2, 2 or 3 times a week; 3 , once a day; 4, a few times a day; and 5, always); (2) amount ("How much urine do you usually leak?"; 0, none; 2 , small amount; 3 , moderate amount; and 4, large amount); (3) impact on QOL ("Overall, how much does leaking urine interfere with your everyday life?"; ranging from 0 [not at all] to 10 [a great deal]); and (4) situations in which UI occurs, which comprised eight items in response to the general question "When does urine leak?" A total ICIQ-SF score ranging from 0 to 21 was calculated by summing the scores of the first three subscales. Severity of UI was categorized into five groups according to patients' total score, as follows: 0 , no symptoms; 1-5, slight; 6-12, moderate; $13-$ 18 , severe; and 19-21, very severe. ${ }^{16)}$

\section{4) Urinary-incontinence-related quality of life}

We assessed UI-related QOL using the third subscale of the ICIQ-SF. For evaluating the impact on QOL, we grouped patients according to whether they had more than 5 points: patients with 5 or more points were categorized as having 'poor QOL,' while patients with 4 points or less were categorized as having 'good QOL.'

\section{5) Geriatric syndromes and related factors}

Geriatric syndromes including cognitive impairment, delirium, depression, ${ }^{7,11,12)}$ and mobility decline, ${ }^{7)}$ as well as factors that are known to be related to $\mathrm{UI},{ }^{17)}$ such as polypharmacy, ${ }^{2)}$ undernutrition, pain, ${ }^{18)}$ and fecal incontinence ${ }^{2)}$ were evaluated with a self-report questionnaire. We used the Korean version of the Ascertain Dementia 8-item Informant Questionnaire to assess cognitive impairment; patients having a score of over 2 points were categorized as exhibiting cognitive 
impairment. ${ }^{19)}$ The Korean version of the Geriatric Depression Scale was used to assess depression; patients who obtained a score of more than 8 points were defined as having depression. Delirium was screened using the Nursing Delirium Screening Scale, with patients scoring more than 2 points on this scale being considered to have delirium. ${ }^{20)}$ Undernutrition was evaluated using the Malnutrition Screening Test, with patients who had more than 2 points being categorized as having undernutrition. Fecal incontinence was assessed via the Low Anterior Resection Syndrome score; patients with over 2 points were classified as having fecal incontinence. Mobility decline, polypharmacy, and pain were each evaluated with a single question: "Can you walk up the stairs without assistance?" for mobility decline; "Are you currently taking five or more medications?" for polypharmacy; ${ }^{21,22)}$ and "Have you had pain more than one day in the last 2 weeks?" for pain. Answering affirmatively to each question indicated the presence of these conditions.

\section{Statistical Analyses}

We used a chi-square test or analysis of covariance to assess general characteristics. To determine if the association of UI with geriatric syndromes and related factors according to gender, multiple logistic regression analysis was carried out after adjusting for confounding factors such as age, length of hospital stay, and past medical history (e.g., stroke, experience of any surgery). Additionally, we conducted another multiple logistic regression analysis to evaluate the associations be- tween geriatric syndromes and UI-related QOL, this time adjusting gender in addition to the confounding variables mentioned above. Statistical analyses were conducted with IBM SPSS statistical software ver. 24.0 (IBM Corp., Armonk, NY, USA). Statistical significance was defined as a $\mathrm{P}$-value of $<0.05$.

\section{RESULTS}

Among 444 patients, 123 reported experiencing UI in the previous 4 weeks. Table 1 shows patients' sociodemographic characteristics. UI patients tended to be older $(77.0 \pm 6.4$ years versus $73.9 \pm 6.4$ years, $\mathrm{P}<0.001)$ and have a longer hospital stay $(9.3 \pm 8.3$ days versus $6.8 \pm 6.1$ days, $\mathrm{P}=0.001)$. More women had experienced UI than men $(13.8 \%$ of women versus $7.0 \%$ of men, $\mathrm{P}<0.001)$.

The frequency, amount, impact on QOL, and severity of UI among the 123 patients with this syndrome are described in Figure 1. Out of all patients with UI, 32.5\% complained of having poor QOL and $22.8 \%$ exhibited severe or very severe UI symptoms. However, the proportions did not significantly differ by gender (Figure 1).

The relationship of UI with geriatric syndromes and related factors by gender are shown in Table 2 . Cognitive impairment (odds ratio [OR], 2.30; 95\% confidence interval [CI], 1.05-5.05), mobility decline (OR, 4.16; 95\% CI, 2.29-7.56), polypharmacy (OR, 3.35; 95\% CI, 1.895.92), fecal incontinence (OR, 3.42; 95\% CI, 1.07-10.93), and pain (OR, $6.80 ; 95 \%$ CI, 3.53-13.09) were all associated with increased probability

Table 1. Baseline characteristics of patients

\begin{tabular}{|c|c|c|c|c|}
\hline Characteristic & Total $(n=444)$ & Normal $(n=321)$ & Urinary incontinence ( $n=123$ ) & P-value \\
\hline Age (y) & $74.8 \pm 6.5$ & $73.9 \pm 6.4$ & $77.0 \pm 6.4$ & $<0.001$ \\
\hline $65-69$ & 50.7 & 57.0 & 34.1 & \\
\hline $70-74$ & 41.7 & 35.8 & 56.9 & \\
\hline$\geq 80$ & 7.7 & 7.2 & 8.9 & \\
\hline Men & 64.2 & 72.6 & 42.3 & $<0.001$ \\
\hline Insurance & & & & 0.915 \\
\hline National health insurance & 93.7 & 93.8 & 93.5 & \\
\hline Medical aid program & 6.3 & 6.2 & 6.5 & \\
\hline Body mass index $\left(\mathrm{kg} / \mathrm{m}^{2}\right)$ & $25.1 \pm 14.3$ & $25.2 \pm 16.4$ & $25.1 \pm 4.2$ & 0.960 \\
\hline Length of stay $(d)$ & $7.5 \pm 6.9$ & $6.8 \pm 6.1$ & $9.3 \pm 8.3$ & 0.001 \\
\hline \multicolumn{5}{|l|}{ Geriatric syndromes } \\
\hline Cognitive impairment & 11.0 & 7.2 & 21.1 & $<0.001$ \\
\hline Depression & 8.1 & 6.9 & 11.4 & 0.118 \\
\hline Delirium & 4.7 & 3.1 & 8.9 & 0.010 \\
\hline Mobility decline & 30.9 & 20.9 & 56.9 & $<0.001$ \\
\hline \multicolumn{5}{|c|}{ Related factors with urinary incontinence } \\
\hline Polypharmacy & 27.5 & 18.1 & 52.0 & $<0.001$ \\
\hline Undernutrition & 11.0 & 8.4 & 17.9 & 0.004 \\
\hline Pain & 20.5 & 9.7 & 48.8 & $<0.001$ \\
\hline Fecal incontinence & 5.4 & 3.4 & 10.6 & 0.003 \\
\hline \multicolumn{5}{|l|}{ Past medical history } \\
\hline Hypertension & 66.1 & 65.3 & 68.2 & 0.625 \\
\hline Diabetes & 36.0 & 33.9 & 42.0 & 0.171 \\
\hline Stroke & 14.4 & 10.2 & 26.1 & $<0.001$ \\
\hline Any surgery & 24.0 & 19.6 & 36.4 & 0.002 \\
\hline
\end{tabular}

Values are presented as mean \pm standard deviation or $\%$. 
of having UI. Among men, delirium (OR, 7.55; 95\% CI, 1.61-35.44) was a significant independent factor not found among women. On the contrary, cognitive impairment was significantly associated with UI in women (OR, 4.19; 95\% CI, 1.14-15.44) but not in men. Fecal incontinence was significantly associated with UI in men, but not in women.

The relationships between geriatric syndromes and UI-related QOL are shown in Table 3. Cognitive impairment, delirium, and mobility decline were not associated with poor QOL. However, depression was significantly associated with poor QOL even after adjusting for age, gender, hospital stay, and past medical history (OR, 8.54; 95\% CI, 1.4351.15).

\section{DISCUSSION}

The prevalence of UI was higher in women than in men; however, UI

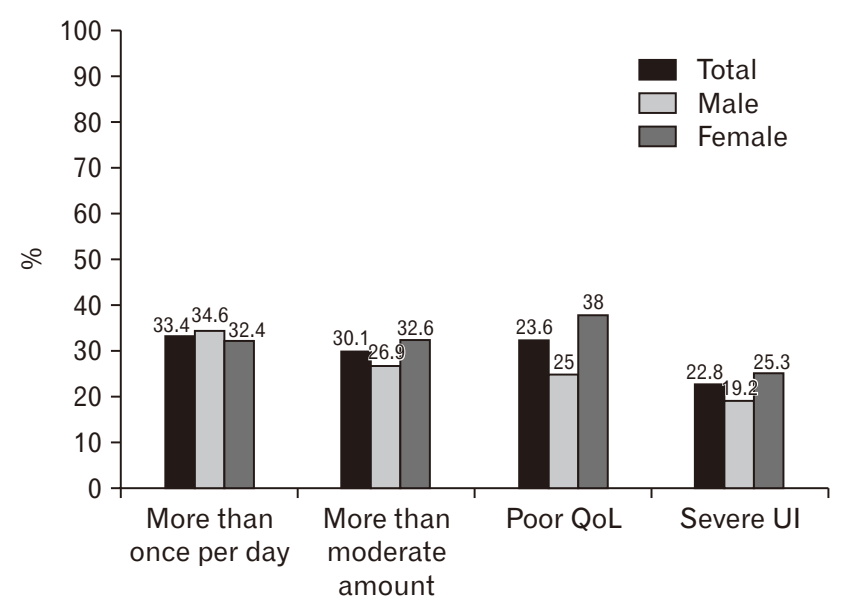

Figure 1. Frequency, amount, severity, and impact on QOL of urinary incontinence (according to the ICIQ-SF). Poor QOL is defined as having more than 5 points on the question "How much does leaking urine interfere with your everyday life?" Severe UI is defined as having more than 13 points on the total ICIQ-SF score. QOL, quality of life; UI, urinary incontinence; ICIQ-SF, International Consultation on Incontinence Questionnaire-Short Form. was only associated with delirium and fecal incontinence in men, whereas cognitive impairment was only associated with UI in women. Mobility decline, polypharmacy, and pain were associated with UI in both genders. The reasons for gender differences are still unclear, but one potential reason might be that men and women experience different types of UI because of the different anatomical structures of their genital organs, and these different types of UI may have distinct causative factors. Past studies on UI among men focused on its onset following surgery of the bladder or prostate; surgeries such as radical prostatectomy are a major risk factor for UI in men, along with prostatic obstruction and fecal incontinence. ${ }^{23)}$ The incidence of delirium might increase after prostate surgery, and medication use-particularly anticholinergic medication for benign prostate hypertrophy-has been recognized as a common precipitating risk factor for delirium. These are all potential explanations for gender differences related to the risk factors of UI. ${ }^{24)}$

We confirmed that the proportion of patients with UI increased with age and that they had longer hospital stays than non-incontinent patients. ${ }^{7)}$ The findings concerning the likelihood of UI increasing with the presence of cognitive impairment was consistent with results from previous studies, even though we used different definitions. ${ }^{25)} \mathrm{Re}$ search on older adults in long-term care settings has shown that impaired functional status can be a predictor of UI, which supports why decreased functional mobility is associated with UI. ${ }^{8)}$ Decreased physical activity might contribute to diminished functional mobility and

Table 3. Association between geriatric syndromes and urinary-incontinence-related quality of life.

\begin{tabular}{lcl}
\hline \multicolumn{1}{c}{ Variable } & Univariate & \multicolumn{1}{c}{ Multivariate $^{*}$} \\
\hline Cognitive impairment & $0.72(0.27-1.87)$ & $0.41(0.11-1.49)$ \\
Depression & $3.21(1.03-9.99)$ & $8.54(1.43-51.15)$ \\
Delirium & $0.43(0.09-2.10)$ & $0.87(0.15-5.07)$ \\
Mobility decline & $0.77(0.36-1.64)$ & $0.68(0.24-1.93)$
\end{tabular}

Values are presented as odds ratio (95\% confidence interval). ${ }^{*}$ Adjusted by age, sex, hospital stay, and past medical history.

Table 2. Association of urinary incontinence with geriatric syndromes and related factors according to sex

\begin{tabular}{|c|c|c|c|c|c|c|}
\hline \multirow{2}{*}{ Variable } & \multicolumn{2}{|c|}{ Total } & \multicolumn{2}{|c|}{ Men } & \multicolumn{2}{|c|}{ Women } \\
\hline & Univariate & Multivariate $^{\star}$ & Univariate & Multivariate* $^{*}$ & Univariate & Multivariate* \\
\hline \multicolumn{7}{|l|}{ Geriatric syndromes } \\
\hline Cognitive impairment & $3.47(1.89-6.37)$ & $2.30(1.05-5.05)$ & $3.41(1.49-7.81)$ & $1.71(0.55-5.32)$ & 3.66 (1.34-10.01) & $4.19(1.14-15.44)$ \\
\hline Depression & $1.75(0.86-3.53)$ & $1.19(0.45-3.14)$ & $1.56(0.59-4.14)$ & $0.91(0.24-3.46)$ & $2.67(0.77-9.25)$ & $1.54(0.30-7.79)$ \\
\hline Delirium & $3.05(1.26-7.39)$ & $2.52(0.82-7.79)$ & $5.95(1.74-20.32)$ & $7.55(1.61-35.44)$ & $1.26(0.35-4.53)$ & $0.82(0.16-4.35)$ \\
\hline Mobility decline & $5.01(3.20-7.83)$ & $4.16(2.29-7.56)$ & $4.28(2.29-8.02)$ & $3.64(1.60-8.28)$ & $7.22(3.45-15.15)$ & $9.01(3.11-26.10)$ \\
\hline \multicolumn{7}{|l|}{$\begin{array}{l}\text { Related factors with urinary } \\
\text { incontinence }\end{array}$} \\
\hline Polypharmacy & $4.92(3.12-7.74)$ & 3.35 (1.89-5.92) & 5.91 (3.09-11.32) & $4.90(2.20-10.90)$ & 3.27 (1.67-6.38) & $2.46(1.03-5.88)$ \\
\hline Undernutrition & $2.37(1.29-4.35)$ & $2.00(0.81-4.96)$ & $3.20(1.45-7.05)$ & $1.81(0.57-5.74)$ & $1.90(0.68-5.27)$ & $3.01(0.59-15.40)$ \\
\hline Pain & $8.91(5.34-14.87)$ & $6.80(3.53-13.09)$ & $5.08(2.47-10.44)$ & $3.73(1.49-9.35)$ & $12.71(5.51-29.34)$ & 15.65 (5.09-48.12) \\
\hline Fecal incontinence & $3.33(1.45-7.65)$ & $3.42(1.07-10.93)$ & 8.29 (2.59-26.53) & $10.15(2.50-41.17)$ & $1.04(0.30-3.54)$ & $0.43(0.06-2.95)$ \\
\hline
\end{tabular}

Values are presented as odds ratio (95\% confidence interval).

*Adjusted by age, hospital stay, and past medical history. 
voiding-related abilities, including muscle strength of the upper and lower extremities, thereby leading to pelvic floor discoordination and UI. ${ }^{26)}$ In agreement with previous studies demonstrating an association between polypharmacy and $\mathrm{UI}^{2}{ }^{26)}$ we also found polypharmacy to be a significant risk factor for increased UI related to adverse drug events, side effects, or drug-drug interactions. ${ }^{1)}$

We further found that depression was associated with poor QOL. Both the odds of UI and poor UI-related QOL increased with the presence of depression, which is consistent with previous research. ${ }^{25,27,28)}$ Our results expand on those of previous studies, however, this study was conducted on hospitalized older patients analyzing the association between depression and direct UI-related QOL using subscales of ICIQ-SF, whereas previous studies focused on health-related QOL in community-dwelling older people. ${ }^{27,28)}$ Many patients are reluctant to seek help or medical treatment because of their perception that UI is untreatable, feelings of shame, or a fear of others knowing their problems. Because of their frequent urination, and feelings of humiliation, incontinent patients tend to avoid social gatherings, visiting friends, travel, and participation in other social activities. Withdrawal from social participation can lead to social isolation, depression, and poor QOL. $^{29)}$

Several limitations of this study should be considered. First, the causal relationship between independent risk factors and UI is uncertain because of the cross-sectional design. Second, this study lacks representativeness because we recruited patients from a single academic hospital. Finally, some misclassifications might have occurred because the diagnosis of UI was based on self-reported data rather than on clinical examinations.

Despite these limitations, our findings are relatively novel and indicate the need for comprehensive clinical interventions to prevent UI in patients with cognitive impairment, mobility decline, polypharmacy, pain, and fecal incontinence. Furthermore, psychosocial support is essential to relieve patients' depressive mood and therefore improve their QOL.

Most older patients admitted to acute care hospitals have at least one geriatric syndrome. UI is a common geriatric syndrome, ${ }^{30)}$ and hospitalized incontinent patients with acute illness often remain in a state of significantly reduced function even after discharge, resulting in a decrease in QOL. ${ }^{11)}$ In conclusion, this study emphasizes the necessity of supporting older incontinent patients with geriatric syndromes in acute care hospital settings. UI patients with geriatric syndromes such as cognitive impairment, depression, mobility decline, polypharmacy, pain, and fecal incontinence require a hospital system that fulfills their clinical needs in a better way and is equipped to identify atrisk patients early during hospitalization. This could be followed by the implementation of specific preventive or curative interventions during and after hospitalization to prevent UI and improve UI-related QOL.

\section{CONFLICT OF INTEREST}

No potential conflict of interest relevant to this article was reported.

\section{ACKNOWLEDGMENTS}

This research was supported by a grant from the Korea Health Technology R\&D Project through the Korea Health Industry Development Institute, funded by the Ministry of Health and Welfare, Republic of Korea (grant no., HI16C0526).

\section{ORCID}

Kyoung Jin Kim: https://orcid.org/0000-0001-9951-5212

Jinyoung Shin: https://orcid.org/0000-0001-9558-1853

Jaekyung Choi: https://orcid.org/0000-0002-0875-7505

Jae-Min Park: https://orcid.org/0000-0001-8873-8832

Hyoung Keun Park: https://orcid.org/0000-0002-7402-8714

Jongmin Lee: https://orcid.org/0000-0001-8718-0099

Seol-Heui Han: https://orcid.org/0000-0003-3608-2514

\section{REFERENCES}

1. Bell SP, Vasilevskis EE, Saraf AA, Jacobsen JM, Kripalani S, Mixon AS, et al. Geriatric syndromes in hospitalized older adults discharged to skilled nursing facilities. J Am Geriatr Soc 2016;64:715-22.

2. Elsawy B, Higgins KE. The geriatric assessment. Am Fam Physician 2011;83:48-56.

3. Hunskaar S, Burgio K, Diokno A, Herzog AR, Hjalmas K, Lapitan MC. Epidemiology and natural history of urinary incontinence in women. Urology 2003;62(4 Suppl 1):16-23.

4. Coyne KS, Sexton CC, Irwin DE, Kopp ZS, Kelleher CJ, Milsom I. The impact of overactive bladder, incontinence and other lower urinary tract symptoms on quality of life, work productivity, sexuality and emotional well-being in men and women: results from the EPIC study. BJU Int 2008;101:1388-95.

5. Milsom I, Coyne KS, Nicholson S, Kvasz M, Chen CI, Wein AJ. Global prevalence and economic burden of urgency urinary incontinence: a systematic review. Eur Urol 2014;65:79-95.

6. Sirls LT, Rashid T. Geriatric urinary incontinence. Geriatr Nephrol Urol 1999;9:87-99.

7. Sier H, Ouslander J, Orzeck S. Urinary incontinence among geriatric patients in an acute-care hospital. JAMA 1987;257:1767-71.

8. Chiu AF, Huang MH, Hsu MH, Liu JL, Chiu JF. Association of urinary incontinence with impaired functional status among older people living in a long-term care setting. Geriatr Gerontol Int 2015;15:296-301.

9. Berardelli M, De Rango F, Morelli M, Corsonello A, Mazzei B, Mari V, et al. Urinary incontinence in the elderly and in the oldest old: correlation with frailty and mortality. Rejuvenation Res 2013;16:206-11.

10. Brown JS, Vittinghoff E, Wyman JF, Stone KL, Nevitt MC, Ensrud KE, et al. Urinary incontinence: does it increase risk for falls and fractures?: study of Osteoporotic Fractures Research Group. J Am Geriatr Soc 2000;48:721-5.

11. Lakhan P, Jones M, Wilson A, Courtney M, Hirdes J, Gray LC. A prospective cohort study of geriatric syndromes among older medical patients admitted to acute care hospitals. J Am Geriatr Soc 2011;59:20018. 
12. Lang PO, Heitz D, Hedelin G, Drame M, Jovenin N, Ankri J, et al. Early markers of prolonged hospital stays in older people: a prospective, multicenter study of 908 inpatients in French acute hospitals. J Am Geriatr Soc 2006;54:1031-9.

13. Silay K, Akinci S, Ulas A, Yalcin A, Silay YS, Akinci MB, et al. Occult urinary incontinence in elderly women and its association with geriatric condition. Eur Rev Med Pharmacol Sci 2016;20:447-51.

14. Avery K, Donovan J, Peters TJ, Shaw C, Gotoh M, Abrams P. ICIQ: a brief and robust measure for evaluating the symptoms and impact of urinary incontinence. Neurourol Urodyn 2004;23:322-30.

15. Abrams P, Cardozo L, Fall M, Griffiths D, Rosier P, Ulmsten U, et al. The standardisation of terminology in lower urinary tract function: report from the standardisation sub-committee of the International Continence Society. Urology 2003;61:37-49.

16. Klovning A, Avery K, Sandvik H, Hunskaar S. Comparison of two questionnaires for assessing the severity of urinary incontinence: the ICIQUI SF versus the incontinence severity index. Neurourol Urodyn 2009; 28:411-5.

17. Kim YS, Han SH, Lee JM, Choi JK, Park JM, Lee KS, et al. 48/6 Model of care for senior clinical care management. Korean J Clin Geriatr 2016; 17:7-15.

18. Figueiredo VF, Amorim JS, Pereira AM, Ferreira PH, Pereira LS. Associations between low back pain, urinary incontinence, and abdominal muscle recruitment as assessed via ultrasonography in the elderly. Braz J Phys Ther 2015;19:70-6.

19. Ryu HJ, Kim HJ, Han SH. Validity and reliability of the Korean version of the AD8 informant interview (K-AD8) in dementia. Alzheimer Dis Assoc Disord 2009;23:371-6.

20. Gaudreau JD, Gagnon P, Harel F, Tremblay A, Roy MA. Fast, systematic, and continuous delirium assessment in hospitalized patients: the nursing delirium screening scale. J Pain Symptom Manage 2005;29: 368-75.

21. Gnjidic D, Hilmer SN, Blyth FM, Naganathan V, Waite L, Seibel MJ, et al. Polypharmacy cutoff and outcomes: five or more medicines were used to identify community-dwelling older men at risk of different adverse outcomes. J Clin Epidemiol 2012;65:989-95.

22. Lim YJ, Kim HY, Choi J, Lee JS, Ahn AL, Oh EJ, et al. Potentially inappropriate medications by beers criteria in older outpatients: prevalence and risk factors. Korean J Fam Med 2016;37:329-33.

23. Linde JM, Nijman RJM, Trzpis M, Broens PMA. Urinary incontinence in the Netherlands: prevalence and associated risk factors in adults. Neurourol Urodyn 2017;36:1519-28.

24. Tognoni P, Simonato A, Robutti N, Pisani M, Cataldi A, Monacelli F, et al. Preoperative risk factors for postoperative delirium (POD) after urological surgery in the elderly. Arch Gerontol Geriatr 2011;52:e1669.

25. Wang CJ, Hung CH, Tang TC, Chen LY, Peng LN, Hsiao FY, et al. Urinary incontinence and its association with frailty among men aged 80 years or older in Taiwan: a cross-sectional study. Rejuvenation Res 2017;20:111-7.

26. Reigota RB, Pedro AO, de Souza Santos Machado V, Costa-Paiva L, Pinto-Neto AM. Prevalence of urinary incontinence and its association with multimorbidity in women aged 50 years or older: a population-based study. Neurourol Urodyn 2016;35:62-8.

27. Takahashi K, Sase E, Kato A, Igari T, Kikuchi K, Jimba M. Psychological resilience and active social participation among older adults with incontinence: a qualitative study. Aging Ment Health 2016;20:1167-73.

28. Hung KJ, Awtrey CS, Tsai AC. Urinary incontinence, depression, and economic outcomes in a cohort of women between the ages of 54 and 65 years. Obstet Gynecol 2014;123:822-7.

29. Goral Turkcu S, Kukulu K. Urinary incontinence and quality of life of women living in nursing homes in the Mediterranean region of Turkey. Psychogeriatrics 2017;17:446-52.

30. Biswas B, Bhattacharyya A, Dasgupta A, Karmakar A, Mallick N, Sembiah S. Urinary incontinence, its risk factors, and quality of life: a study among women aged 50 years and above in a rural health facility of West Bengal. J Midlife Health 2017;8:130-6. 